\title{
Mode of Action of Datemycin on the Growth of Candida Albicans
}

\section{Agglutination Reaction of Candida Albicans Observed}

in the Presence of Datemycin

By

\section{Shozo Nakajima}

(中島 主蔵)

From the Department of Bacteriology, School of Medicine, Tohoku

University, Sendai. Director: Prof. M. Kuroya

(Received for publication, April 11, 1959)

A little is known on the agglutination phenomena of any microorganisms with some antibiotics. Gros ${ }^{11}$ was the first who reported in 1949 on the agglutination of several gram positive and also negative bacteria with streptomycin. Later on, in 1950, Lantterade and others ${ }^{2,}$ have noticed the agglutination of Sta. albus and E. coli with polymyxin.

Datemycin $^{3)}$, an antibiotic possessing a limited inhibitory activity to a certain species of Candida have been isolated in this laboratory from a culture filtrate of a species of $B$. subtilis, M-14. When the crude substance of the antibiotic dissolved in phosphate buffer was shaken at $37^{\circ} \mathrm{C}$ with a suspension of Candida albicans, characteristic agglutination pattern have been found. Further studies on the site of action of datemycin in a slide cell culture system, revealed some interesting result suggesting the mechanism of the agglutination. In fact, mycelium formation of Candida albicans under the existence of this antibiotic was the real mode of this agglutination reaction. The result obtained along this line is the subject of this communication.

\section{EXPERIMENTAL}

Materials and Methods

\section{Datemycin}

Active principle in culture filtrate of $B$. subtilis (M-14) was once adsorbed to charcoal at $\mathrm{pH} 7.0$ and eluted by $80 \%$ acetone at acidic $\mathrm{pH}$. Further fractionation of the eluate was performed on the column of $\mathrm{Al}_{2} \mathrm{O}_{3}$. The active fraction was collected and dried in vacuo. Dried material was once dissolved in methanol and precipitated with acetone. Thus the crude datemycin was obtained. Further purification was performed by extracting the aqueous solution of datemycin with butanol containing 2 
per cent $p$-toluensulfonic acid. From this butanol extract, the agent was again transferred to aqueous layer at $\mathrm{pH}$ 7.0. From this aqueous solution, $p$-toluensulfonic acid was removed by passing the fluid through the column of Amberlite IRA 411 (Cl type). After this procedure, purified preparation was obtained, though not crystallized. Crude datemycin was positive in both Fehling and ninhydrin test and had around the half activity of purified preparation. The latter was positive only in ninhydrin test and inhibited the growth of $\mathrm{C}$. albicans in a concentration of $6 \mathrm{mcg} / \mathrm{ml}$.

\section{Candida}

Eight strains of Candida albicans, i.e. M-2, M-8, M-9, M-10, Harvard, Takeda, FIA-1001, and Duke was used for this study. In addition, each one strain of C. tropicalis, G. pseudotropicalis, C. krusei, C. parakrusei, G. guilliermondi was also used. All these strains have been propagated on Sabouraud's medium at $37^{\circ} \mathrm{C}$ for 48 hours. Then they were collected by centrifugation, washed three times with phosphate buffer of $\mathrm{pH} 7.0$ and suspended again in the same buffer. The concentration of the suspension was standardized turbidimetrically. Final concentration of these cells thus adjusted was $1.3 \times 10^{7} / \mathrm{ml}$, corresponding to the wet weight of $2 \mathrm{mg} / \mathrm{ml}$.

Agglutination test

Usually M-9 strain of $C$. albicans was used for this purpose, unless otherwise noted. Datemycin was dissolved in phosphate buffer of $\mathrm{pH}$ 7.0 and to $1 \mathrm{ml}$ aliquots of this solution, equal amount of the cell suspension described above, was added. These tubes were usually shaken at $37^{\circ} \mathrm{C}$ for 4 hours and again left at room temperature until next morning. The result was read only macroscopically at the beginning of this study, but microscopical examination was also paralleled later. Usually the positive reaction became apparent after three hours of incubation but reached to the maximum after four hours. However, if these series of tubes were left overnight at room temperature, reading of the result was very easy. The concentration of the cell ranging from $0.5 \mathrm{mg}$ to $2 \mathrm{mg}$ did not affect this result seriously. The agglutination pattern here observed was rather flocculous than granular and only after repeated shaking of the tubes, homogenous turbidity was obtainable (Fig. 1).

Slide cell culture technique

According to the description of Nickerson, ${ }^{4)}$ slide cell culture of serveral Candidae was performed to test the site of action of this antibiotic. Basal medium contained $1.0 \mathrm{~g}$ of $\left(\mathrm{NH}_{4}\right)_{2} \mathrm{SO}_{4}, 1 \mathrm{~g}$ of $\mathrm{KH}_{2} \mathrm{PO}_{4}, 5.0 \mathrm{mcg}$ of biotin, $15 \mathrm{~g}$ of agar and $20 \mathrm{~g}$ of soluble starch. They were dissolved in $1,000 \mathrm{ml}$ of distilled water and $0.5 \mathrm{ml}$ aliquot of the medium was spread on a slide glass in a circle of $1.5 \times 2.0 \mathrm{~cm}^{2}$. One loopful organisms taken from Sabouraud's slant was inoculated on the surface of this medium. The slide was closed by cover slips as usual and incubated at $27^{\circ} \mathrm{C}$. After two 
days of incubation, the mycelium formation and several changes which occurred in the cell was examined microscopically. In this medium, the excellent outgrowth of mycelium was followed with all of the strains tested, after 2 days of incubation. However, when 0.5 per cent of glucose or $M$ / 1,000 of cystein was added to this medium, the formation of mycelium was completely inhibited.

\section{Results}

\section{Limited activity of this antibiotics on a species Candida albicans}

As have been reported elsewhere ${ }^{3)}$, the antibiotic had an extremely limited range of activity, when the usual test organisms were examined for their susceptibility. One mg. of this antibiotic did not inhibit all tested bacteria both gram positive and negative. Neighter fungi were susceptible. However, when several species of Candida were tested, $C$. albicans was fairly susceptible to this antibiotic. One of the result obtained with purified preparation was shown in Table I. In this study, the activity

\section{TABLE I}

Effect of Datemycin on the Growth of Several Species of Candida

\begin{tabular}{|c|c|c|c|}
\hline \multirow{2}{*}{ Candida } & \multirow{2}{*}{ tested } & \multicolumn{2}{|c|}{$\begin{array}{l}\text { Minimal inhibition concentration } \\
(\mathrm{mcg} / \mathrm{ml}) \text { obtained by }\end{array}$} \\
\hline & & $\begin{array}{l}\text { Agar streak dilution } \\
\text { method* }\end{array}$ & Broth dilution method* \\
\hline Candia albicans & $\mathbf{M}-2$ & 16 & 4 \\
\hline$" \quad \quad "$ & M-8 & 32 & 4 \\
\hline$"$ & M-9 & 125 & 8 \\
\hline$"$ & $M-10$ & 125 & 8 \\
\hline$"$ & Harvard & 16 & 8 \\
\hline C. krisei & & 500 & 63 \\
\hline C. parakrusei & & $>1000$ & 125 \\
\hline C. tropicalis & & $>1000$ & 250 \\
\hline C. stellatoidea & & 125 & 63 \\
\hline
\end{tabular}

* Sabouraud's agar media and Sabouraud's broth were used.

was tested both by means of agar streak plate method and broth dilution method, where the activity was shown higher in the latter procedure. Thus all tested strains of $C$. albicans were susceptible. But the other species of Candida were not so susceptible as albicans. All of other species of Saccharomyces tested so far, were not inhibited with this antibiotic, though the result was omitted from the Table.

The specifity of the agglutination with regard to the tested organisms 
The agglutinability of several strains described above was tested with this antibiotic. The preparation here used was a crude one. A typical result obtained from repeated experiments was illustrated in Table II.

TABLE II

Agglutination of Candida in the Presence of Datemycin

\begin{tabular}{|c|c|c|c|c|c|c|c|c|c|c|c|}
\hline \multirow{2}{*}{\multicolumn{2}{|c|}{ Candida tested }} & \multicolumn{10}{|c|}{ Concentration of datemycin $(\mathrm{mcg} / \mathrm{ml})$} \\
\hline & & 1000 & 500 & 250 & 125 & 63 & 32 & 16 & 8 & 4 & 0 \\
\hline \multirow[t]{8}{*}{ C. albicans } & $M-2$ & H* $^{*}$ & \# & H & + & - & - & - & - & - & - \\
\hline & $M-8$ & 世 & \# & $H$ & + & - & - & - & - & - & - \\
\hline & M-9 & 世 & H & H & + & - & - & - & - & - & - \\
\hline & $\mathbf{M}-10$ & H & 世 & $H$ & + & - & - & - & - & - & - \\
\hline & Takeda & \# & 世 & H & $H$ & + & - & - & - & - & - \\
\hline & FIA 1001 & H & H & $H$ & + & - & - & - & - & - & - \\
\hline & Duke & 世 & $H$ & + & \pm & - & - & - & - & - & - \\
\hline & Harvard & H & $H$ & $H$ & $H$ & $H$ & + & - & - & - & - \\
\hline \multicolumn{2}{|c|}{ C. tropicalis } & - & - & - & - & - & - & - & - & - & - \\
\hline \multicolumn{2}{|c|}{ C. pseudotropicalis } & - & - & - & - & - & - & - & - & - & - \\
\hline \multicolumn{2}{|c|}{ C. krusei } & - & - & - & - & - & - & - & - & - & - \\
\hline \multicolumn{2}{|c|}{ C. parakursei } & - & - & - & - & - & - & - & - & - & - \\
\hline \multicolumn{2}{|c|}{ C. guilliermondi } & - & - & - & - & - & - & - & - & - & - \\
\hline \multicolumn{2}{|c|}{ C. stellatoidea } & - & - & - & - & - & - & - & - & - & - \\
\hline
\end{tabular}

All tested strains of $G$. albicans were agglutinated. But the other species of Candida which are known to be fairly resistant to this antibiotic as has been already shown in Table I, was not agglutinated at all. Thus the correlation was found between the agglutinability and sensitivity of the strain to this antibiotic. Several kind of gram negative and positive bacteria, which are already known to be resistant to this antibiotic were also tested for their agglutinability. They all gave the negative result.

One more remark to be noticed here is the fact, that when killed M-9 strain obtained after boiling for 10 minutes was tested for their agglutinability, the result was completely negative.

Optimum $\mathrm{pH}$ for this reaction was also tested with M-9. The result was shown in Table III and the highest titer was obtained at $\mathrm{pH} 7.0$.

Optimum temperature for this reaction was also examined. When these tubes were left in an ice box all the way through after mixing the antibiotic with cell suspension, no agglutination patterns were found. The titer obtained at $37^{\circ} \mathrm{C}$ was not quite different from that obtained at $27^{\circ} \mathrm{G}$. 
TABLE III

Optimum $\mathrm{pH}$ for the Agglutination

\begin{tabular}{|c|c|c|c|c|c|c|c|c|}
\hline \multirow{2}{*}{$\mathrm{pH}$} & \multicolumn{8}{|c|}{ Concentration of datemycin $(\mathrm{mcg} / \mathrm{ml})$} \\
\hline & 5000 & 2500 & 1250 & 630 & 320 & 160 & 80 & 0 \\
\hline 4.0 & H* $^{*}$ & H & H & H & + & - & - & - \\
\hline 6.0 & \# & H & W & H & + & - & - & - \\
\hline 7.0 & H & \# & H & $H$ & + & \pm & - & - \\
\hline 8.0 & H & H & H & $H$ & + & - & - & - \\
\hline 8.3 & W & H & $H$ & + & - & - & - & - \\
\hline
\end{tabular}

Effeci of sugars on the agglutination

On the final purification procedure of the antibiotic, the agglutination test was conducted in parallel with the inhibition test. Unexpected result here obtained was that the final preparation described in the section of materials and methods did not agglutinate M-9 at all. However, it was already known at that occasion that the final preparation of the antibiotic did not contaminated with Fehling positive substances, as has been pointed out before. Thus the participation of some sugars in this agglutination test have been suggested. In order to confirm the above idea, the agglutination reaction was examined with final preparation of datemycin but with and without glucose. As a result, apparent agglutination occurred only in the presence of glucose. Concentration effect of glucose was shown in Table IV.

As a result the concentration more than $0.01 \%$ was required for the stimulation of the reaction.

Other sugars than glucose were also tested for their effect on the agglutination. Among them, sucrose and galactose had the same activity as glucose, but only the weak reaction was obtained in the presence of mannose. Lactose, however, did not have this kind of activity at all (Table IV).

One has to notice here that Candida albicans are already known to ferment glucose, surcrose and galactose but not mannose and lactose, and the fact was also confirmed in my own hand. Thus the stimulation effect of sugars on the agglutination reaction was found only with sugars which are known to be fermented with this strain.

Microscopic examination of the agglutination reaction

The characteristics of the agglutination reaction described above will be summarized briefly as follows. Firstly, the reaction seems to be temperature dependent and the optimum $\mathrm{pH}$ for the reaction is 7.0. Secondly, only the susceptible cells to this antibiotic were agglutinated in the vegetative 
TABLE IV

Effect of Sugars on the Agglutination of Candida albicans M-9 in the Presence of Purified Datemycin

\begin{tabular}{|c|c|c|}
\hline Tested sugars & Concentration & Agglutination \\
\hline Glucose & $1 \%$ & $H^{*}$ \\
\hline$"$ & $0.1 \%$ & $H$ \\
\hline$"$ & $0.01 \%$ & $\mathrm{HH}$ \\
\hline$"$ & $0.001 \%$ & - \\
\hline Mannose & $1 \%$ & \pm \\
\hline Galactose & $1 \%$ & H \\
\hline Lactose & $1 \%$ & - \\
\hline Sucrose & $1 \%$ & \# \\
\hline Control & & - \\
\hline
\end{tabular}

state but not in killed form. Thirdly, only sugars which are utilized with these cells had the stimulating action on the agglutination. Thus the agglutination reaction here observed seems to be correlated rather to the growth of the tested strains than to be a known physical phenomenon.

For this reason, the agglutination pattern obtained in the test tubes were examined microscopically.

In the control tube, all of the cells were in yeast form after the incubation of 24 hours at $37^{\circ} \mathrm{C}$. No characteristic change was found inside the cell. However, when $1 \mathrm{mg}$ of datemycin was contained in the same system, the number of elongated cells first increased (See Fig. 1). This increase was detectable as early as two hours after the incubation. After 6 hours, macro-visible agglutination was found and the microscopic examination revealed that the cells are all in the form of mycelium. Thus the consistent change from yeast cell to elongated cell, and from elongated cell to the mycelium was established with all of the $C$. albicans tested in the presence of datemycin.

Mycelium formation in the slide cell culture

Mycelium formation described above was further examined in detail in the slide cell culture system.

The basal medium here used allowed the excellent mycelium formation of all the tested organisms but the addition of glucose in 0.5 per cent concentration inhibited the reaction completely. However, when datemycin was added in the medium in addition to $0.5 \%$ glucose, mycelium formation was again observed with Candida such as M-2, M-8 and M-9 (see Table V). At that time, the optimal concentration of datemycin to get the mycelium formation was between 2 to $8 \mathrm{mcg}$ in the presence of 


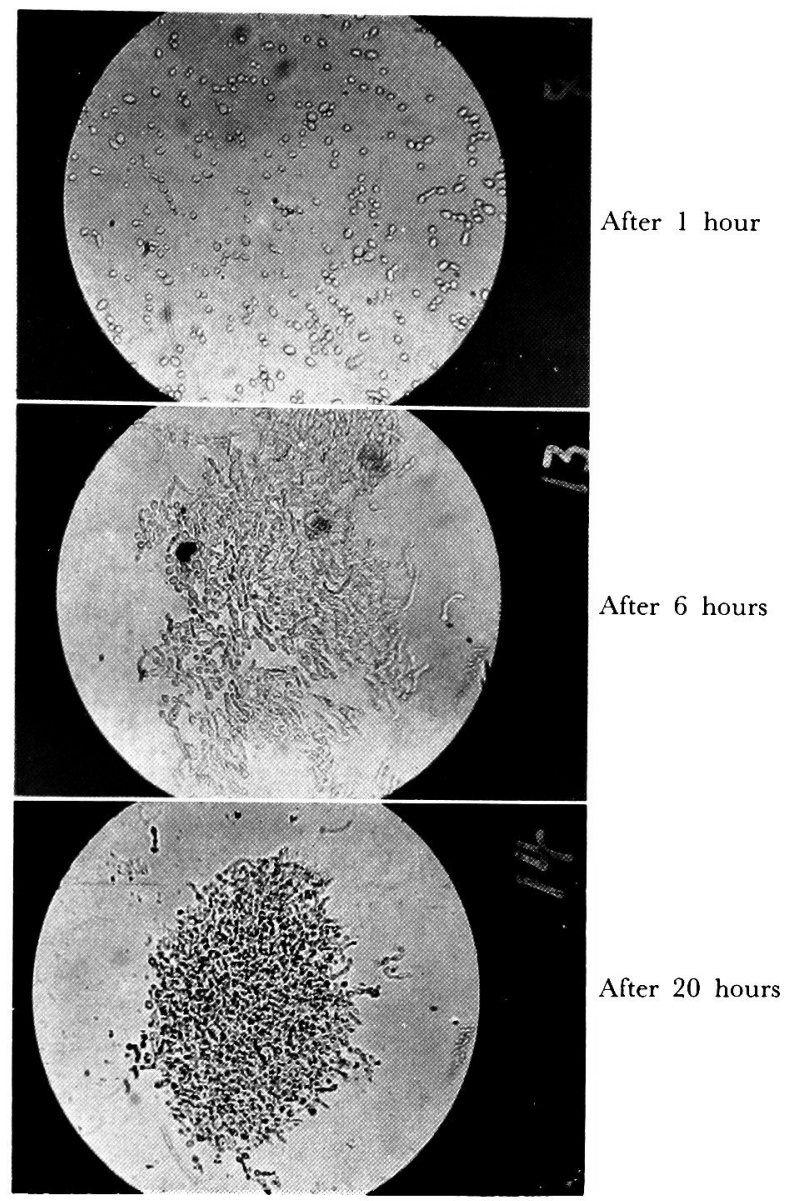

Fig. 1. Agglutination of C. albicans in the presence of datemycin.

0.5 per cent glucose. The higher concentration of the antibiotic, however, showed the toxic effect on the cell. The degeneration of the cell occurred first and mycelium formation did not follow.

When $C$. tropicalis and $C$. krusei which were known to be resistant to this antibiotic were used as test-organism, the mycelium formation was also found but only with higher concentration of the antibiotic such as 125 to $1,000 \mathrm{mcg}$. Within this concentrations, however, the degenerative change on the cell was not recognized.

In addition to glucose, the effect of cysteine on the mycelium formation was also tested. When M-8 strain was used for this purpose, though $M / 10,000$ of cystein did not affect the formation of the mycelium, $M / 1,000$ concentration inhibited the formation completely. Further, the effect of 
TABLE V

Mycelium Formation Found in the Presence of Datemycin in the Medium Containing 0.5\% Glucose

\begin{tabular}{|c|c|c|c|c|c|c|c|c|c|c|c|c|}
\hline \multirow{3}{*}{$\begin{array}{l}\text { Concentration } \\
\text { of datemycin } \\
(\mathrm{mcg} / \mathrm{ml})\end{array}$} & \multicolumn{12}{|c|}{ Tested candidae } \\
\hline & \multicolumn{2}{|c|}{$\mathrm{M}-2$} & \multicolumn{2}{|c|}{$\mathrm{M}-8$} & \multicolumn{2}{|c|}{$M-9$} & \multicolumn{2}{|c|}{ Takeda } & \multicolumn{2}{|c|}{ C. trop } & \multicolumn{2}{|c|}{ C. kruse } \\
\hline & $\mathrm{m}^{*}$ & $\mathrm{D} \dagger$ & $\mathrm{m}$ & $\mathrm{D}$ & $\mathrm{m}$ & D & $\mathrm{m}$ & $\mathrm{D}$ & $\mathrm{m}$ & $\mathrm{D}$ & $\mathbf{m}$ & D \\
\hline 1000 & - & $(\mathrm{HH})$ & - & (H) & - & $(H)$ & - & (H) & $H$ & & $H$ & \\
\hline 125 & - & $(W)$ & - & $(\mathrm{H})$ & - & (姍) & - & (H) & H & & + & \\
\hline 32 & - & $(H)$ & - & $(H)$ & H & & - & $(+)$ & - & $(-)$ & - & $(-)$ \\
\hline 8 & H & & H & & Ht & & 卅 & & - & $(-)$ & - & $(-)$ \\
\hline 2 & $H$ & & - & $(-)$ & - & $(-)$ & - & $(-)$ & - & $(-)$ & - & $(-)$ \\
\hline 0 & - & $(-)$ & - & $(-)$ & - & $(-)$ & - & $(-)$ & - & $(-)$ & - & $(-)$ \\
\hline
\end{tabular}

* Grade of mycelium formation. + Grade of degencration of cells.

\section{TABLE VI}

Effect of Datemycin on the Growth of $C$. albicans M-8 in the Medium Containing Different Concentration of Cysteine

\begin{tabular}{|c|c|c|c|c|c|c|c|c|c|c|c|c|c|c|}
\hline \multirow{3}{*}{ 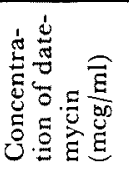 } & \multicolumn{14}{|c|}{ Concentration of cysteine } \\
\hline & \multicolumn{2}{|c|}{$1 / 100 \mathrm{M}$} & \multicolumn{2}{|c|}{$1 / 300 \mathrm{M}$} & \multicolumn{2}{|c|}{$1 / 500 M$} & \multicolumn{2}{|c|}{$1 / 800 \mathrm{M}$} & \multicolumn{2}{|c|}{$1 / 1,000 \mathrm{M}$} & \multicolumn{2}{|c|}{$1 / 10,000 \mathrm{M}$} & \multicolumn{2}{|c|}{0} \\
\hline & $\mathrm{m}^{*}$ & $\mathrm{D} \dagger$ & $\mathrm{m}$ & $\mathrm{D}$ & $\mathrm{m}$ & $\mathrm{D}$ & $\mathrm{m}$ & $\mathrm{D}$ & $\mathrm{m}$ & $\mathrm{D}$ & $\mathrm{m}$ & $\mathrm{D}$ & $\mathrm{m}$ & $\mathrm{D}$ \\
\hline 1000 & - & $(-)$ & - & $(-)$ & - & $(+)$ & - & $(H)$ & - & $(\mathrm{HH})$ & - & $(\mathrm{HH})$ & - & (H) \\
\hline 500 & - & $(-)$ & - & $(-)$ & - & $(-)$ & - & $(H)$ & - & (H) & - & $(+H)$ & - & (H) \\
\hline 250 & - & $(-)$ & - & $(-)$ & - & $(-)$ & - & $(\mathrm{H})$ & - & (H) & - & (井) & - & (H) \\
\hline 125 & - & $(-)$ & - & $(-)$ & - & $(-)$ & - & $(H)$ & - & $(\mathrm{HH})$ & - & (H) & - & (H) \\
\hline 63 & - & $(-)$ & - & $(-)$ & - & $(-)$ & - & $(+)$ & - & $(H)$ & - & $(H)$ & - & (H) \\
\hline 32 & - & $(-)$ & - & $(-)$ & - & $(-)$ & - & $(+)$ & - & (H) & - & (H) & - & (H) \\
\hline 16 & - & $(-)$ & - & $(-)$ & - & $(-)$ & - & $(-)$ & $H$ & & - & (HI) & - & (H) \\
\hline 8 & - & $(-)$ & - & $(-)$ & - & $(-)$ & - & $(-)$ & 世 & & H & & - & (H) \\
\hline 4 & - & $(-)$ & - & $(-)$ & - & $(-)$ & - & $(-)$ & H & & H & & - & $(H)$ \\
\hline 2 & - & $(-)$ & - & $(-)$ & - & $(-)$ & - & $(-)$ & - & $(-)$ & $H$ & & H & \\
\hline 1 & - & $(-)$ & - & $(-)$ & - & $(-)$ & - & $(-)$ & - & $(-)$ & HH & & H & \\
\hline 0.5 & - & $(-)$ & - & $(-)$ & - & $(-)$ & - & $(-)$ & - & $(-)$ & H & & $H$ & \\
\hline 0 & - & $(-)$ & - & $(-)$ & - & $(-)$ & - & $(-)$ & - & $(-)$ & HW & & 册 & \\
\hline
\end{tabular}

* Grade of mycelium formation. † Grade of degeneration of cells.

datemycin on the cell growing in the presence of different concentration of cysteine was examined (see, Table VI). The formation of mycelium with M-8 was inhibited with $M / 1,000$ cysteine, but the addition of datemycin ranging from 4 to $8 \mathrm{mcg}$ allowed the formation of mycelium. The higher 
concentration of datemycin, such as $32 \mathrm{mcg}$ again induced the degeneration of the cells. This degenerative effect was found with $4 \mathrm{mcg}$ concentration of the antibiotic without addition of cysteine. Thus the interesting result here obtained, is the competitive effect of cysteine on the activity of datemycin. For example, when the concentration of cystein was shifted to $M / 500$, only slight degeneration was obtained at the concentration as high as $1 \mathrm{mg}$ per $\mathrm{ml}$ of datemycin but not at the concentration of $32 \mathrm{mcg}$. If the concentration of cystein was $M / 300$, even $1 \mathrm{mg}$ of datemycin did not affect the state of cell at all. On the basis of this observation, the competitative effect of cystein on the inhibitory activity of datemycin was tested later. In the presence of $M / 100$ of cystein, the concentration of datemycin inhibiting the growth of M-9, dropped from $2 \mathrm{mcg}$ to $50 \mathrm{mcg}$.

For comparative study, $C$. kursei, known as resistent to this antibiotic was also tested in the same manner (see Table VII). In the presence of

\section{TABLE VII}

Effect of Datemycin on the Growth of C. krusei in the Medium Containing Different Concentration of Cysteine

\begin{tabular}{|c|c|c|c|c|c|c|c|c|c|c|}
\hline \multirow{3}{*}{$\begin{array}{l}\text { Concentration } \\
\text { of datemycin } \\
(\mathrm{mcg} / \mathrm{ml})\end{array}$} & \multicolumn{10}{|c|}{ Concentration of cysteine } \\
\hline & \multicolumn{2}{|c|}{$1 / 100 M$} & \multicolumn{2}{|c|}{$1 / 500 M$} & \multicolumn{2}{|c|}{$1 / 1000 \mathrm{M}$} & \multicolumn{2}{|c|}{$1 / 10000 M$} & \multicolumn{2}{|c|}{$\mathrm{D}$} \\
\hline & $\mathrm{m}^{*}$ & $\mathrm{D} \dagger$ & $\mathrm{m}$ & $\mathrm{D}$ & $\mathrm{m}$ & $\mathrm{D}$ & $\mathrm{m}$ & $\mathrm{D}$ & $\mathrm{m}$ & $\mathrm{D}$ \\
\hline 1000 & - & $(-)$ & H & & 世 & & H & & H & \\
\hline 500 & - & $(-)$ & H & & H & & $H$ & & H & \\
\hline 250 & - & $(-)$ & 世 & & \# & & 卅 & & Ht & \\
\hline 125 & - & $(-)$ & + & $(-)$ & H & & H & & \# & \\
\hline 63 & - & $(-)$ & - & $(-)$ & H & & $H$ & & H & \\
\hline 32 & - & $(-)$ & - & $(-)$ & H & & \# & & H & \\
\hline 16 & - & $(-)$ & - & $(-)$ & $H$ & & 世 & & 世 & \\
\hline 8 & - & $(-)$ & - & $(-)$ & + & & $\mathrm{Ht}$ & & HIt & \\
\hline 4 & - & $(-)$ & - & $(-)$ & - & $(-)$ & H & & H & \\
\hline 2 & - & $(-)$ & - & $(-)$ & - & $(-)$ & H & & Ht & \\
\hline 1 & - & $(-)$ & - & $(-)$ & - & $(-)$ & H & & Ht & \\
\hline 0.5 & - & $(-)$ & - & $(-)$ & - & $(-)$ & H & & $H$ & \\
\hline 0 & - & $(-)$ & - & $(-)$ & - & $(-)$ & $H$ & & m & \\
\hline
\end{tabular}

* $\mathrm{m}$ grade of mycelium formation. $\quad+\mathrm{D}$ grade of degeneration of cells.

$M / 1,000$ of cysteine, $8 \mathrm{mcg}$ or more concentration of datemycin allowed the mycelium formation. In increasing the concentration of cysteine, mycelium formation was found only in the presence of high concentration of datemycin. Thus the activity of stimulating the mycelium formation was shown also with this fairly resistant strain, but in a little bit higher 
concentration.

As far as the degenerative effect is concerned, however, even in the absence of cysteine, no effect was found on C. kursei with $1 \mathrm{mg}$ of datemycin. This was quite different from the result obtained with susceptible strains.

\section{Discussion}

As has been pointed out in the preface of this communication, two papers $^{1 / 2)}$ have already appeared on the agglutination reaction of bacteria caused by the addition of antibiotics. Two antibiotics, streptomycin and polymyxin, which has the basic character in nature reacted with the RNA on the surface of gram-positive bacteria and formed an insoluble complex. Thus the agglutination phenomena have been elucidated in these cases.

In this case, however, the site of agglutination was quite different. It was the mycelium formation of Candida by the addition of an antibiotic, datemycin. The optimum $\mathrm{pH}$ of the reaction was 7.0 and the reaction required the temperature. Only susceptible cells of Candida were agglutinated with lower concentration of the antibiotic. But these cells have to be in an alive state, because heat killed organisms were not agglutinated. The addition of some sugars, such as glucose, which were fermented by this organism were necessary for this agglutination. The addition of cystein, which is known to inhibit the formation of mycelium ${ }^{5 / 6}$ ) inhibited this agglutination. Thus all of the circumstantial evidence suggest that the mycelium formation is a site of this agglutination and the idea was confirmed by microscopic examination as has been illustrated in Fig. 1 .

The action of datemycin was reversed by cysteine both in the inhibition test and in the mycelium formation as was the case with penicillin and streptomycin. Stimulative activity of the antibiotic in the formation of mycelium will be correlated to this fact, if one assume that the inhibition of the $\mathrm{SH}$-protein synthesis is correlated to the formation of elongated cell(5)78). The experimental result which was not described here indicates that the sensitive strain to this antibiotic contains lower concentration of SH-compound when compared to that of resistant strain.

In spite of the fact that cysteine reversed the action of this antibiotic, the higher inhibitory activity of datemycin was obtained in a fluid culture system rather than in an aerobic condition, such as on the surface of the agar. This observation holds true if one compare the concentration of datemycin, which cause the agglutination in test tubes and the concentration wh ch cause the mycelium formation in a closed slide cell culture system. In the former test at least $25 \mathrm{mcg}$ was necessary to cause the agglutination of M-8 but in the latter test as low as $4 \mathrm{mcg}$ of the antibiotic stimulated the mycelium formation. Rather the concentration of the 
organisms tested in these systems or the nutritional condition of the medium should be taken into account for these discrepancies.

\section{SUMMARY}

In the presence of an antibiotic, datemycin, the agglutination phenomenon of $G$. albicans was first recognized. Further study revealed that the agglutination reaction was restricted to only susceptible strains, and these cells have to be in vegetative state but not killed. The reaction was evident only in room or blood temperature but not in the cold. The optimum $\mathrm{pH}$ was in the vicinities of 7.0. Sugars which are utilized by the tested organisms had the stimulating effect on the agglutination. Further microscopic examination revealed that the mycelium formation of the tested cells was the real mode of this agglutination.

In a slide cell culture system, the fact that the lower concentration of datemycin stimulates the mycelium formation of Candida was quite evident. Both agglutinative and inhibitory activities of datemycin was partly reversed by the addition of cysteine.

\section{References}

1) Gros, F., Ann. Inst. Pasteur, 1949, 77, 246.

2) Lantterade, C. \& Mechehoeuf, M., Ann. Inst. Pasteur, 1950, 78, 751.

3) Chiba, T. \& Nakajima, S., Chemotherapy, 1957, 5, 182.

4) Nickerson, W. J., J. Infect. Dis., 1953, 92, 21.

5) Nickerson, W.J., Biochem. Biophys. Acta, 1949, 3, 46.

6) Salvin, S. B., J. Infect. Dis., 1949, 84, 275.

7) Nickerson, W.J., Nature, 1948, 162, 241.

8) Rapkine, L., Ann. physiol. physiochim. biol., 1931, 7, 383. 